\title{
Bilateral jugular veins occlusion in mice: Clinical and histological findings
}

\author{
Greco $A^{1,2}$, Auletta $L^{3}$, Coda $A^{1}$, Baldi $A^{4,5}$, Montanaro $D^{4}$, Albanese $S^{1}$, Pappatà $S^{1}$ and $M_{a n c i n i} M^{1 *}$ \\ ${ }^{1}$ Istituto di Biostrutture e Bioimmagini, CNR, Napoli, Italy \\ ${ }^{2}$ Interdepartmental Center of Veterinary Radiology, University of Naples Federico II, Naples 80137, Italy \\ ${ }^{3}$ IRCCS SDN, Napoli, Italy \\ ${ }^{4}$ Ceinge, Biotecnologie Avanzate, Scarl, Napoli, Italy \\ ${ }^{5}$ Dipartimento di Scienze e Tecnologie Ambientali, Biologiche e Farmaceutiche, Università della Campania “L. Vanvitelli”, Caserta, Italy
}

\begin{abstract}
An impaired venous drainage from the brain and spinal cord as result of outflow obstruction in the extracranial venous system was considered as a possible contributing factor to the pathogenesis and clinical manifestations of neurodegenerative diseases.

The aim of the study was to determine the contribution of impaired jugular veins to the status of the brain tissues insufficiently drained by extracranial veins. Thirtyeight mice were used for our experiments, 22 mice underwent to the bilateral ligation of the internal and external Jugular veins "(ligated group)", 16 mice underwent to the same operative procedures and postoperative precautions but without jugular veins ligation "(sham-operated group)". Following the surgery, mice were monitored every day for the development of clinical symptoms. After 20 days and 3 months post-surgery, mice were sacrificed. Each brain was cut out sagittal to divide the two hemispheres. The samples were subjected to both histological and immunohistochemically staining using slides to define edema, inflammatory reaction, iron and fibrinogen deposit and demyelination.
\end{abstract}

Of the 22 ligated group 7 showed a Grade 1 of clinical impairment. Histopathological assays showed the presence of brain edema and micro-hemorrhages. A significant higher number of positive inflammatory cells in the brains of ligated Group in comparison to sham group ( $\mathrm{p}<0.05$ ) was detected. No evidence of demyelination nor iron or fibrin deposition in Ligated group was demonstrated.

In conclusion, jugular vein ligation altered cerebral hemodynamics without causing significant neurological symptoms. The outflow obstruction in the extracranial venous system in rodent models is able to elicit a mild inflammatory process.

\begin{abstract}
Abbreviations: EJV: External jugular veins; IJV: Internal jugular veins; LG: Ligated group; SG: Sham group; SALG: Sub-acute ligated group; CLG: Chronic ligated group; SASG: Sub-acute sham group; CSG: Chronic sham group; HFUS: High frequency ultrasound; US: Ultrasound; CBF: Cerebral blood flow.
\end{abstract}

\section{Introduction}

It was hypothesized that an alteration of venous outflow could be associated to many diseases [1-11]. An alteration of the venous outflow could determine an intracranial venous congestion, a blood brain barrier destruction, and a deposition of iron and macromolecules that could induce an inflammatory status, an immune response and a peripheral macrophages recruitment [12]. An increase in the calcium levels in the internal jugular vein, mainly localized in the tunica adventitia of micro vessels [13], and a focal thickening of the vein wall correlated with a higher expression type II collagen [14] were demonstrated in MS patients, suggesting that biochemical and morphological alterations may occur in the vein wall of some of patients. Furthermore, different methods of quantification, such as catheter venography, conventional Doppler ultrasonography (US), contrast enhanced US, plethysmography and MRI venography, have demonstrated the presence of a venous obstruction in these patients [15-17]. On the other hand, other studies failed to demonstrate a significant difference in the venous cerebral and cervical outflows in MS patients compared to controls [18-21]. Moreover, it was demonstrated that fibrinogen deposition in the extravascular space could determine an activation of the immunity against myelin and focal inflammatory activation [22]. The development of animal models of cerebral veins obstructions might contribute to better evaluate and understand the effects of chronic cerebral outflow obstruction on functional, biochemical and morphological alterations of brain tissues. To the best of our knowledge, only two papers described the effect of ligation of neck veins in mice $[23,24]$, but these studies took in consideration only the ligature of the external Jugular veins. Interestingly, a recent study demonstrated that also the internal Jugular vein is an important drainage route for venous flow in mice [25]. Recently, we have developed a mice model of cerebral blood outflow obstruction obtained through the bilateral ligation of the internal and external jugular veins [26] that might reproduce intracerebral venous stasis similar to that observed in patients with internal jugular vein obstruction.

${ }^{\star}$ Correspondence to: Marcello Mancini, Istituto di Biostrutture e Bioimmagini, CNR, Napoli, Italy, E-mail: marcello.mancini58@gmail.com

Key words: neurodegenerative diseases, animal models, jugular vein obstruction, venous hypertension

Received: December 17, 2019; Accepted: January 03, 2020; Published: January 06,2020 


\section{Material and methods}

\section{Animals}

The animal protocols used in this work were approved by the Animal Use and Ethic Committee of CEINGE, Biotecnologie Avanzate, Naples Italy and the Ministry of Italyn Health (Protocol 257/2018-PR). All experiments were performed in accordance with Federation of European Laboratory Animal Science Associations and the European Communities Council directive $(2010 / 63 / \mathrm{EU})$ guidelines. Thirtyeight (18female/20male) 8-12 weeks-old C57/black mice were used for our experiments, 22 mice underwent to the bilateral ligation of the internal (IJV) and external Jugular veins (EJV) ("ligated group" LG), 16 mice underwent to the same operative procedures and postoperative precautions but without jugular veins ligation ("sham-operated group" SG).

\section{Surgery}

All surgeries were performed in general anesthesia, with 5\% isoflurane (Iso-Vet $1000 \mathrm{mg} / \mathrm{g}$ Inhalation Vapor, Piramal Healthcare UK Ltd., Northumberland, UK) in oxygen $(2 \mathrm{~L} / \mathrm{min})$; the analgesic protocol consisted in one intraperitoneal injections every $12 \mathrm{~h}$ of tramadol hydrochloride (Altadol $50 \mathrm{mg} / \mathrm{ml}$, Formevet Spa, Milan, Italy) at $30 \mathrm{mg} / \mathrm{kg}$.

Mice were placed in dorsal recumbency on a heated pad. After a midline incision of the ventral portion of the neck, the salivary glands were disposed dorsally to visualize and ligate the EJV. After the dissection of the muscles underling the trachea, it was possible to expose and ligate the IJV [18] (Figures 1A,1B and 1C). Eleven mice of LG group were sacrificed 20 days post-surgery to explore subacute effects (Sub-Acute ligated group SALG) and the remaining 11 were sacrificed after 3 months to explore chronic effects of IJV/EJV obstruction (Chronic ligated group CLG). At the same way, 8 sham mice were sacrificed after 20 days (Sub-acute sham group SASG), and 8 sham mice were sacrificed after 3 months (Chronic sham group CSG).

High Frequency Ultrasound (HFUS) was used to confirm the effectiveness of the IJVs and EJVs (Figures 2A, 2B) occlusion after surgery in the LG and to control the patency in the sham operated mice. Following the surgery, mice were monitored every day for the development of clinical symptoms according to the Grading system used for clinical assessment of experimental encephalomyelitis (EAE) according to Miller et al. [27].

\section{Animal sacrifice, perfusion and brain fixation}

After 20 days and 3 months post-surgery, mice were sacrificed. Animals were deeply anaesthetized and were placed in the supine position. An abdominal incision was made with surgical scissors to expose the heart. Through a peristaltic pump a needle was insert into the apex of the left ventricle and immediately after a cut was made on the right atrium. Animals received about $30 \mathrm{~mL}$ ice-cold phosphatebuffered saline (0.1 M PBS), until the fluid exiting the right atrium was entirely clear, followed by about $60 \mathrm{~mL}$ of $4 \%$ paraformaldehyde in saline solution. After perfusion of mice was complete, brains were isolated and post-fixed overnight at $4^{\circ} \mathrm{C}$ in $4 \%$ paraformaldehyde [28].

\section{Histology and immunohistochemistry}

Each brain was cut out sagittally to divide the two hemispheres. The processing was performed with the Bio-Optica VTP 300 processor. Then, the samples were included in paraffin. The cuts were performed with the Leica RM 2245 microtome. For each sample a serial cutting of 10 slides was made. Three sections of $5 \mu \mathrm{m}$ each were placed on each slide. The samples were subjected to both histological and immunohistochemical staining using slides in most cases immediately consecutive to define edema, inflammatory reaction, iron and fibrinogen deposit and demyelinization.

Histology: Hematoxylin/eosin staining: after the de-icing followed by the decreasing scale of ethanol's, the samples were stained with hematoxylin for $5 \mathrm{~min}$ and then with eosin for $10 \mathrm{sec}$, and after the passage in a scale of ethanol with increasing concentration, the slides were mounted. This coloring allowed the morphological evaluation.

Immunohistochemistry: The Abcam anti myelin ab40390 antibody (final dilution 1.400) was used to highlight myelin and the Abcam anti neuro filament ab8135 antibody was used (final dilution 1:1000) to detect neurofilaments. The unmasking was performed with the pH6 citrate buffer of Dako (code S2369) in a thermostatic bath at $97^{\circ} \mathrm{C}$ for 20 minutes. The detection system used was the polymer of the Dako K5001. The Biorad rat anti-mouse F4/80 biotilinate MCA497B antibody [final dilution 1:200] was used to highlight the presence of macrophages. The unmasking was performed with the pH6 citrate buffer of Dako (code S2369)in thermostatic bath at $97^{\circ} \mathrm{C}$ for 20 minutes. The detection system used was the kit Scy Tek Laboratories ultraTek. Quantitative analysis of macrophages was performed in all animal of each experimental groups. To this aim, we have generated a score of MCA497B antibody staining by averaging the total number of positive cells assessed in 5 different cerebral areas close to a vessel. For the

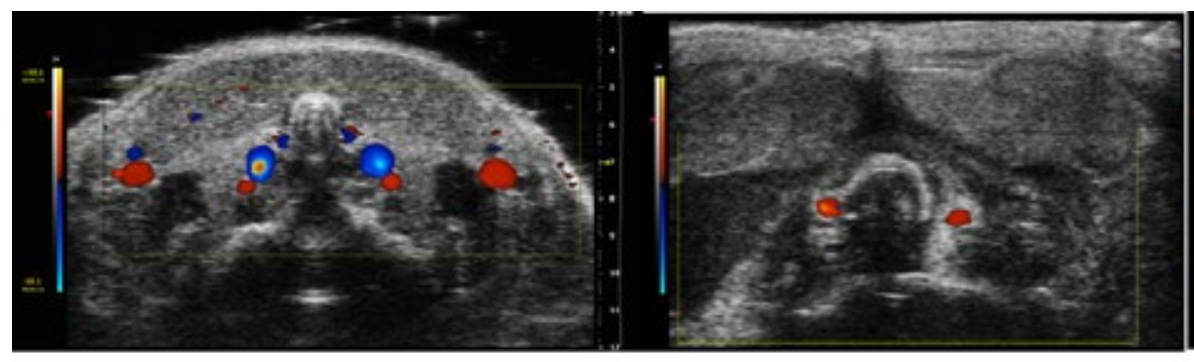

A
B

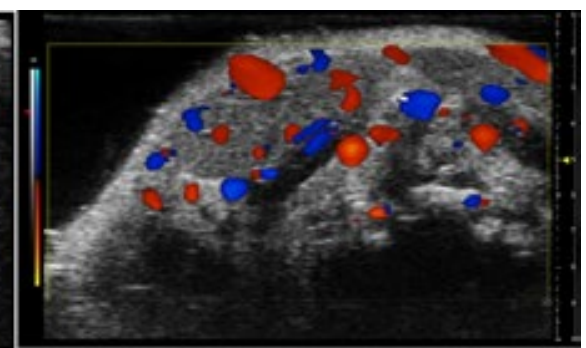

C

Figure 1. Ultrasound imaging. HFUS imaging of the neck was performed with a dedicated equipment system for small animal imaging (VEVO 2100 , FUJIFILM VisualSonics, Inc., Toronto, Ontario, Canada), in general anesthesia as previously described. Color Doppler images with a $20 \mathrm{MHz}$ probe (MS250; FUJIFILM VisualSonics, Inc., Toronto, Ontario, Canada) was done to explore the blood flow in the internal and external jugular veins before surgery (A) and two days after surgery (B). After surgery it is not evident bood flow in the internal and in the external Jugular vein. It is possible to notice flow only in the carotid artery. C About 15 days no evidence of flow in IJV and EJV after surgery while collateral veins are present in the mouse neck 

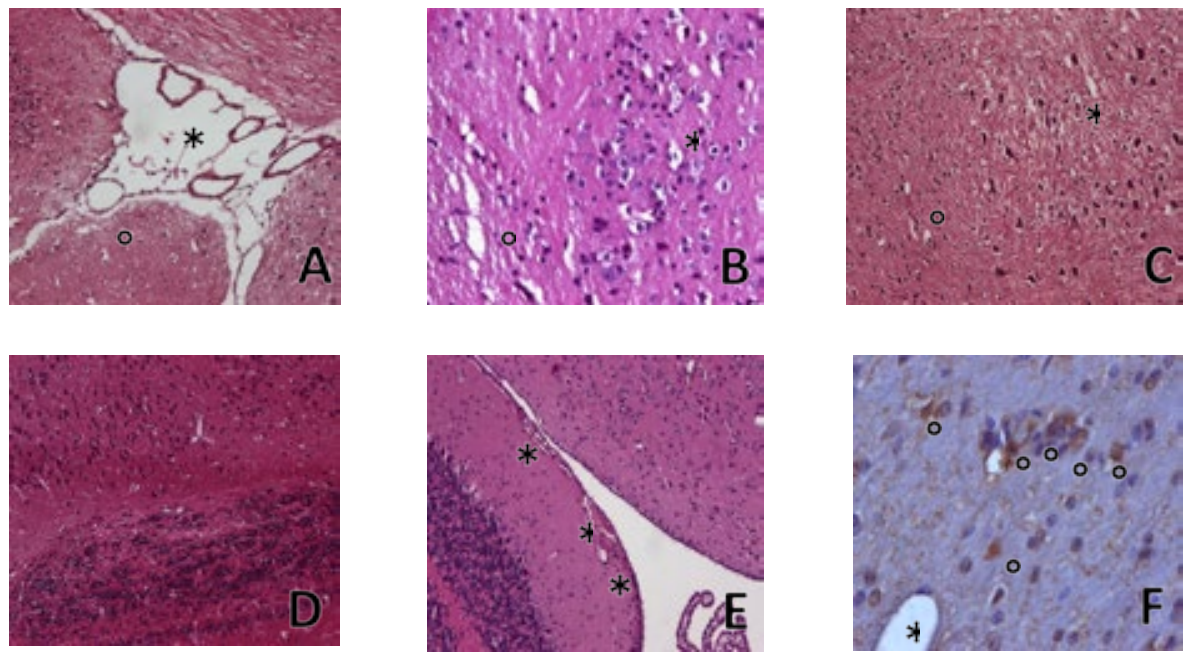

Figure 2. Histology and immunohistochemistry.

A) Floor of the IV ventricle with choroid plexus residues with clear intra-parenchymal $\left(^{\circ}\right)$ and meningeal $(*)$ edema in an animal of the SALG group. Hematoxylin and Eosin staining (original magnification 10x)

B) Numerous cortical neurons with intra-parenchymal edema at peri-cellular $\left(^{\circ}\right)$ and peri-vascular $(*)$ level in an animal of the SALG group. Hematoxylin and Eosin staining (original magnification 20x)

C) Numerous cortical neurons with intra-parenchymal (frontal cortex) edema at peri-cellular $\left(^{\circ}\right)$ and peri-vascular $(*)$ level in an animal treated with the "chronic" protocol. Hematoxylin and Eosin staining (original magnification 10x)

D) An area of cerebellar cortex of a SASG animal shows a mild edema. Hematoxylin and Eosin staining (original magnification X10)

E) Sub-ependymal micro-hemorrhages $(*)$ at the level of the IV ventricle of a SALG, Hematoxylin and Eosin staining (original magnification 10x)

F) A small group of activated microglial cells $\left(^{\circ}\right)$ near a cerebral vessel $(*)$ in the cortex of a mouse of the SALG group, Immunohistochemistry for MCA497B antibody (original magnification 20x)

statistical analysis, the median number of positive cells for each brain observed was used and compared in the different groups of animals.

Perls staining (Bio-Optica kit code 04-180807) was used for the detection of iron deposits. Fibrinogen was evidenced in brain tissue samples with an anti $\alpha$ chain fibrinogen antibody (1:500; ABcam 19079). Unmasking was performed in $10 \mathrm{mM}$ citrate buffer, $\mathrm{pH}$ 6, for $45 \mathrm{~min}$ at $97^{\circ} \mathrm{C}$; blocking was performed with BSA $2,5 \%$, normal goat serum $0.05 \%$, PBS $1 \%$, Tween $0,5 \%$ for $1 \mathrm{~h}$ at room temperature. The signal was revealed according to DAKO kits, for $15 \mathrm{~min}$ [each, for biotin and streptavidin], at room temperature. DAB was from DakoCytomation, and the slides were mounted and examined under a Leica DC500 compound microscope (Nussloch, Germany).

\section{Statistical analysis}

Neurological score was evaluated for all animals participating to the study and the results were expressed as mean \pm SD. Nonparametric test U di Mann-Withney (median and range) was used to compare LG and SG in sub-acute and in chronic stages. Results was considered significant at $\mathrm{p}<0,05$.

\section{Results}

\section{Surgical procedure}

Thirty-eight mice were submitted to the surgical procedure. All mice of the SG survived during surgery procedure and the whole trial period. Of the 22 LG mice, 18 survived (four mice died immediately after surgery due to intraoperative complications), and in two mice the bilateral ligation of EJV and IJV was unsuccessful for an incorrect surgical ligation of the external Jugular veins and post-surgical bleeding. Finally, 8 mice of the SALG and 8 mice of the CLG, and all mice of the SG underwent to clinical evaluation and histological analysis.
All mice underwent to HFUS before and after the surgical procedure (Figures 1A and $1 \mathrm{~B}$ ) that demonstrated the absence of the flow in all operated IJVs and EJVs mice except the 2 with unsuccessful ligation. In 5/22 mice of the LG, the induction of collateral veins was demonstrable in the neck, 10-15 days after surgery (Figure 1C).

\section{Clinical evaluation}

All mice presented a weight loss of $10 \%$, one week after surgery, but recovered in few days. No change in the breathing and in the social behavior was observed after surgery.

Regarding the locomotion, and according to the classification of the EAE used for comparison, all the sham mice were considered Grade 0 , showing no clinical signs at all. Of the 22 operated mice, $7 / 22$ showed a loss of tail tonicity and of ability of curling the tail, or/and mild hind limb weakness (Grade 1). These symptoms were present in 4/11 mice of the SALG and in 3/11 mice of the CLG. The other mice did not present any neurological sign.

\section{Histology}

Histopathological assays showed in the LG (Figures 2A and 2C), but not in the SG (Figure 2D), the presence of brain edema and microhemorrhages (Figure 2E). We did not notice a significant difference between the brains of mice that underwent to bilateral ligation of the internal and external jugular veins at 20 days and 3 months postsurgery, excluding a more diffuse edema in the sub-acute (SALG) group than in the chronic (CLG) groups. Neither, a specific topography for the highlighted lesions was detected in the examined areas (cerebral cortex, cerebellum, brain stem until IV ventricle). Edema was visible both at the parenchymal and at the meningeal level, while micro-hemorrhages were detected essentially at the meningeal level (Figure 2E). 
The presence of macrophage/microglia infiltration was also investigated by means of immunohistochemical analysis with MCA497B antibody, a specific marker for macrophages and activated microglia. We found a significant higher number of positive inflammatory cells in the brains of treated animals near the cerebral veins respect to the shams. The number of macrophages per field was significantly higher in SALG compared to SASG (median 4.5, range 3-6 and median 1, range $0-2$, respectively, $\mathrm{p}<0.05$ ) as well as in CLG compared to CSG (median 4 , range $3-5$, and median 1 , range $0-2$, respectively, $\mathrm{p}<0.05)$. The number of macrophages was not significantly different between SALG and CLG. In Figure 2F a small group of macrophages, near a cerebral vein of a treated animal is depicted. We did not observe the typical macrophage/microglia infiltration present in human multiple sclerosis as well as experimental murine models of demyelinating disease, we found only few isolated CD68 cells or isolated on in small groups. No evidence of demyelination in the brains of LG respect to the SG (data not shown) was demonstrated, in either sub-acute or chronic stages. Similar negative findings were observed for iron or fibrin deposition.

\section{Discussion}

We have studied a mice model of cerebral outflow obstruction obtained with bilateral legation of the internal and external jugular veins, in order to evaluate the effects of venous stasis on brain tissue. Venous obstruction determines an activation of an injury cascade and an inflammatory response [29]. A difference between LG and SG was observed in the cerebral edema, micro hemorrhages and inflammation while histopathology did not show any evidence of iron deposition and demyelination in the brain.

Diffuse abnormal accumulation of fluid in the brain parenchyma secondary to obstruction of the jugular veins was found in all LG animals in the subacute stage and it was still present, although to a lesser extent, in the more chronic stages.

Fibrinogen is a major component in the blood that is considered a marker of BBB disruption [30]. The absence of plasma components as fibrinogen, red blood cells and lymphocytes in perivenular space suggest that the BBB is intact in our LG animals. Therefore, venular hypertension do not induce vasogenic edema but only passive interstitial edema in brain tissue. An increase of the venous pressure could reduce the capillary blood flow velocity, the micro vessel perfusion and the permeability possibly resulting in a passive diffusion through the $\mathrm{BBB}$ of water and a wide range of lipid-soluble molecules, that are bloodflow dependent. An alteration in veins and venules pressure induces a modification of neurovascular units more than in arterioles or capillaries [29-33].

Some authors suggest that veins changes could have a central role in disease pathogenesis rather than representing a secondary effect of neuroinflammation or neurodegeneration $[34,35]$. The use of Ultrahigh-field MRI scanners improve definition of lesion morphology and the association with vasculature [36-38]. The study of intra parenchymal venous system, obtained by the use of $\mathrm{T} 2^{*}$-weighted magnitude and phase imaging confirms results of pathological studies showing that many multiple sclerosis plaques are found around the venules $[39,40]$. Moreover, the investigation of blood barrier abnormalities in MS at 7.0T or $3.0 \mathrm{~T}$ showed that most enhancing lesions have a centrifugal pattern of enhancement suggesting that grow outwards from the central vein $[41,42]$.

Multiple micro hemorrhages were also detected. Increase in intracranial venous pressure might have caused brain swelling and meningeal capillary damage leading to micro-hemorrhage [43]. The leptomeningeal location of micro-hemorrhages might be also related to a primary impact of the increased central venous pressure on the meninges, the main central venous discharge. This kind of micro hemorrhages could explain the inflammatory infiltration in the meningeal space and adjacent perivascular space in type 2 cortical lesions of gray matter that are often localized around blood vessels and developed independently of white matter lesions [44].

We also found a mild but significantly increase in the number of CD68 positive cells isolated or in small groups in LG as compared to SG in both sub-acute and chronic stages, in particular located around venous vessels, suggesting the occurrence of mild inflammatory response.

These findings are in part different from those reported in mouse [23] and rat [24] models of chronic bilateral external jugular vein ligation (EJVL), showing no evidence of significant cerebral edema, micro-hemorrages or microglial/macrophage activation. Atkinson et al. failed to reveal any evidence of edema, BBB alteration and inflammatory cell infiltration such as activated neutrophils, macrophages, and microglia as measured in vivo with myeloperoxidase-gadolinium (MPOGd) and MRI and in vitro with flow cytometric analysis and immunohistochemitry in the brain and perivascular space of SJL mice studied between 4 and 6 months after JVL. Similarly, no microglial/ macrophage nor lymphocytes could be detected in either perivascular or brain parenchymal in JVL rats studied at 8 months after surgery. Interestingly increased activated astrocytes were found at GFAP staining in white matter regions in the EJVL group as compared to the shamoperated group [24]. In addition, these authors also reported marked increase in iron deposition mainly located in white matter tracts within oligodendrocytes and in the endothelial lining of the blood vessels as well as mild brain tissue edema surrounding the mildly enlarged perivascular Virchow Robin spaces in JVL rats. In our study and in that of Atkinson, astrocytes activation was not assessed while we failed to find cerebral or vascular/ perivascular iron deposition. Discrepancies between our results and those previously reported might be related to differences in the severity of JVL models, rodents used (mice or rats), mice strains (female SJL and C57/black mice), rodents age at surgery (812 weeks old in SJL and C57/black mice, 6 months in rats), times from surgery ( 20 days to 8 months). Of particular relevance is the JVL model used. Our model of bilateral occlusion of both external and internal jugular veins is expected to result in more severe chronic cerebral venous insufficiency than that induced by the bilateral occlusion of external jugular veins. It was demonstrated that the intracranial venous outflow runs in three directions: into the IJV and EJVs and into the vertebral venous plexus. Therefore, the only interruption of EJV at the neck level does not allow complete interruption of cerebral drainage with many possibilities of extra-intracranial collaterals can compensate the venous hypertension and the effects on brain tissue therefore could be minimal [25].

In line with previous findings [23-24] we did not find signs of demyelination or major neurological symptoms after surgery.

The mice with mild cerebral edema were all completely asymptomatic. This may be due to the fact that obstruction of neck veins is not able to increase the intracranial pressure to the level that produces a significant decrease in Cerebral Blood Flow both by decreasing effective perfusion pressure and by compressing cerebral vessel. However, chronic edema could produce a silent and progressive activation of inflammatory process and tissue damage. Other factors are needed to induce significant blood brain barrier breakdown 
with macromolecular and/or peripheral inflammatory cells brain infiltration, major microglial/macrophages activation, demyelination, axonal damage and clinical signs, the typical hallmarks of human multiple sclerosis and of Experimental autoimmune encephalomyelitis (EAE) rodent models of multiples sclerosis. It could be hypothesized that changes in brain neurovascular unit and cerebral blood flow or a combination of both may be an early sign of disease prior than other typical neuro-inflammatory and neurodegenerative events would occur.

\section{Limitations}

\section{This study has some limitations}

The mouse is an imperfect model for studying the human cerebral venous circulation, since there are differences between mouse and human vascular anatomy $[17,25]$, and the neck veins are always at the same level of the heart.

\section{We have not measured cerebral venous pressure.}

We have only indirect signs of BBB integrity because in our study we have not measured the permeability of BBB.

Despite these limitations, in conclusion, the animal model that was developed in this study could be useful in research on the role of cerebral vein in the pathogenesis of neurodegenerative disorders and to investigate complex and multifactorial diseases such as Multiple Sclerosis for which a single mouse model cannot capture and adequately incorporate all the clinical, radiological, pathological, genetic and immunological features of the disease.

\section{Funding}

This work was supported by a research grant from the Italian Ministry for Education University and Research in the framework of PRIN (2010XE5L2R_004). The funders had no role in study design, data collection and analysis, decision to publish, or preparation of the manuscript.

\section{Acknowledgments}

The authors thank Dr. Stefania Daniele and Dr. Monica Ragucci for the editing of the manuscript.

\section{References}

1. Zamboni P, Galeotti R, Menegatti E (2009) Chronic cerebrospinal venous insufficiency in patients with multiple sclerosis. J Neurol Neurosurg Psychiatry 80: 392-399.

2. Zivadinov R, Poloni GU, Marr K (2011) Decreased brain venous vasculature visibility on susceptibility-weighted imaging venography in patients with multiple sclerosis is related to chronic cerebrospinal venous insufficiency. BMC Neurol 11: 128.

3. Liu M, Xu H, Wang Y (2015) Patterns of chronic venous insufficiency in the dural sinuses and extracranial draining veins and their relationship with white matter hyperintensities for patients with Parkinson's disease. J Vasc Surg 61: 1511-1520.

4. Chung CP, Beggs C, Wang PN (2014) Jugular venous reflux and white matter abnormalities in Alzheimer's disease: A pilot study. J Alzheimers Dis 39: 601-609.

5. Alpini D, Bavera PM, Di Berardino F (2016) Bridging the gap between chronic cerebrospinal venous insufficiency and Me'nie're disease. Veins Lymphatics 5: 5687

6. Bruno A, Napolitano M, Califano L (2017) The prevalence of chronic cerebrospinal venous insufficiency in Meniere disease: 24-Month follow-up after angioplasty. Vasc Interv Radiol 28: 388-391.

7. Sander D, Winbeck K, Etgen T (2000) Disturbance of venous flow patterns in patients with transient global amnesia. Lancet 356: 1982-1984.

8. Jonas J, Paques M, Monés J (2010) Retinal vein occlusions. Dev Ophthalmol 47: 111135
9. Nicholson P, Brinjikji W, Radovanovic I (2018) Venous sinus stenting for idiopathic intracranial hypertension: a systematic review and meta-analysis. Neurointerv Surg.

10. Fofi L, Giugni E, Vadalà R (2012) Cerebral transverse sinus morphology as detected by MR venography in patients with chronic migraine. Headache 52: 1254-1261.

11. Chung C-PC, Hu HH (2010) Pathogenesis of leukoaraiosis: Role of jugular venous reflux. MedHypotheses 75: 85-90

12. Haacke E M, Garbern J, Miao Y (2010) Iron stores and cerebral veins in ms studied by susceptibility weighted imaging. Int Angiol 29: 149-157.

13. Pascolo L, Gianoncelli A, Riccardi C (2014) Calcium microdepositions in jugular truncular venous malformations revealed by Synchrotron-based XRF imaging. Sci Rep 4: 6540

14. Coen M, Menegatti E, Salvi F (2013) Altered collagen expression in jugular veins in multiple sclerosis. Cardiovasc Pathol 22: 33-38.

15. Mancini M, Morra VB, Di Donato O (2012) Multiple sclerosis: cerebral circulation time. Radiology 262: 947-955.

16. Haacke EM, Makki M, Ge Y (2009) Characterizing iron deposition in multiple sclerosis lesions using susceptibility weighted imaging. J Magn Reson Imaging 29: 537-544.

17. Karmon Y, Zivadinov R, Weinstock-Guttman B (2013) Comparison of intravascular ultrasound with conventional venography for detection of extracranial venous abnormalities indicative of chronic cerebrospinal venous insufficiency. $J$ Vasc Interv Radiol 24: 1487-1498.

18. Doepp F, Paul F, Valdueza J M (2010) No cerebrocervical venous congestion in patients with multiple sclerosis. Ann Neurol 68: 173-183.

19. Sundstrom P, Wahlin A, Ambarki K (2010) Venous and cerebrospinal fluid flow in multiple sclerosis: a case-control study. Ann Neurol 68: 255-259.

20. Wattjes MP, van Oosten BW, de Graaf WL (2011) No association of abnormal cranial venous drainage with multiple sclerosis: a magnetic resonance venography and flowquantification study. J Neurol Neurosurg Psychiatry 82: 429-435.

21. Yamout B, Herlopian A, Issa Z (2010) Extracranial venous stenosis is an unlikely cause of multiple sclerosis. Mult Scler 16: 1341-1348.

22. Ryu JK, Petersen MA, Murray SG (2015) Blood coagulation protein fibrinogen promotes autoimmunity and demyelination via chemokine release and antigen presentation. Nat Commun 6: 8164.

23. Atkinson W, Forghani R, Wojtkiewicz GR (2012) Ligation of the jugular veins does not result in brain inflammation or demyelination in mice. PlosOne 7: e33671

24. Zakaria MMA, Mikhael SY, Hussein AKA (2017) Failure of the vascular hypothesis of multiple sclerosis in a rat model of chronic cerebrospinal venous insufficiency. Folia Neuropathol 55: 49-59.

25. Mancini M, Greco A, Tedeschi E (2015) Head and neck veins of the mouse. A magnetic resonance,micro computed tomography and high frequency color doppler ultrasound study. PLoSOne 10: e0129912

26. Auletta L, Greco A, Albanese S (2017) Original Research: Feasibility and safety of two surgical techniques for the development of an animal model of jugular vein occlusion. Exp Biol Med (Maywood) 242: 22-28.

27. Miller SD, Karpus WJ (2007) Experimental autoimmune encephalomyelitis in the mouse. Curr Protoc Immunol 15: 1

28. Gage GJ, Kipke DR, Shain W (2012) Whole animal perfusion fixation for rodents. $J$ Vis $\operatorname{Exp} 65: 3564$.

29. Bedford THB, Burgess KR, Johnson P (1935) The effect of increased intracrania venous pressure on the pressure of the cerberospinal fluid. Brain 58: 429-447.

30. Ferris EB (1939) The effect of high intracranial venous pressure upon the cerebral circulation and its relation to cerebral symptoms. J Clin Invest 18: 19-24.

31. Raisis JE, Kindt GW, McGillicuddy JE (1979) The effects of primary elevation of cerebral venous pressure on cerebral hemodynamics and intracranial pressure. $J$ Surg Res 26: 101-107

32. Cuypers J, Matakas F, Potolicchio SJ (1976) Effect of central venous pressure on brain tissue pressure and brain volume. J Neurosurg 45: 89-94.

33. Schoser BG, Riemenschneider N, Hansen HC (1999) The impact of raised intracranial pressure on cerebral venous hemodynamics: a prospective venous transcranial Doppler ultrasonography study. $J$ Neurosurg 91: 744-749.

34. Spencer JI, Bell JS, DeLuca GC (2018) Vascular pathology in multiple sclerosis: reframing pathogenesis around the blood-brain barrier. J Neurol Neurosurg Psychiatry 89: $42-52$. 
35. Haacke EM, Beggs CB, Habib C (2012) The role of venous abnormalities in neurological disease. Reviews on Recent Clinical Trials 7: 100

36. Tallantyre EC, Brookes MJ, Dixon JE (2008) Demonstrating the perivascular distribution of MS lesions in vivo with 7-Tesla MRI. Neurology 70: 2076-2078.

37. Kilsdonk ID, Lopez-Soriano A, Kuijer JP (2014) Morphological features of MS lesions on FLAIR* at 7 T and their relation to patient characteristics. J Neurol 261: 1356-1364.

38. Ge Y, Zohrabian VM, Grossman RI. Seven-Tesla (2008) Magnetic resonance imaging: New vision of microvascular abnormalities in multiple sclerosis. Arch Neurol 65: 812-816.

39. Hammond KE, Lupo JM, Xu D (2007) Development of a robust method for generating 7.0 T multichannel phase images of the brain with application to normal volunteers and patients with neurological diseases. Neuroimage 39: 1682-1692.
40. Mainero C, Benner T, Radding A (2009) In vivo imaging of cortical pathology in multiple sclerosis using ultra-high field MRI. Neurology 73: 941-948.

41. Tallantyre EC, Dixon JE, Donaldson I (2011) Ultra-high-field imaging distinguishes MS lesions from asymptomatic white matter lesions. Neurology 76: 534-539.

42. Gaitán MI, Sati P, Inati SJ (2013) Initial investigation of the blood-brain barrier in MS lesions at 7 TESLA. Mult Scler 19: 1068-10673.

43. Absinta M, Sati P, Gaitán MI (2013) Seven-tesla phase imaging of acute multiple sclerosis lesions: a new window into the inflammatory process. Ann Neurol 74: 669-78.

44. Calabrese M, Castellaro M. Cortical (2017) Gray matter MR imaging in multiple sclerosis. Neuroimaging Clinics of North America 27: 301-312.

Copyright: (C2020 Greco A. This is an open-access article distributed under the terms of the Creative Commons Attribution License, which permits unrestricted use, distribution, and reproduction in any medium, provided the original author and source are credited. 